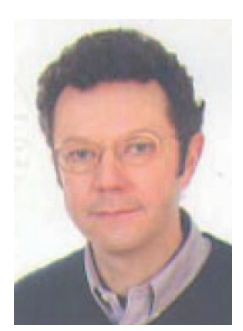

"Notwithstanding prolonged suppression of viral load, approximately $15-30 \%$ of antiretroviral-treated subjects lack CD4 T-cell increase."

\title{
Stefano Rusconi
}

Dipartimento di Scienze Biomediche e Cliniche (DIBIC) "Luigi Sacco", Divisione Clinicizzata di Malattie Infettive, Università degli Studi di Milano; Italy = Tel. : +39 0239042949 = Fax: +39 0250319758

nstefano.rusconi@unimi.it

HIV infection produces immune activation [1,2], and it is important to remember that HIV is the causative agent of chronic viral infection. This activation leads to immune deficiency through the destruction of target cells, and also induces some other pernicious consequences, such as low thymic output, lymphoid tissue fibrosis and $\mathrm{T}$ - and B-cell dysfunction. Other more long-term effects are represented by inflammation, tissue damage and coagulopathy, including an increase in non-AIDS morbidity and mortality [3].

This is what the natural history of HIV would be without antiretroviral (ARV) drugs, which target several steps in the HIV lifecycle. ARV treatment promotes lymphocyte redistribution via multiple phenomena: a biphasic increase in circulating CD4 T cells [4] and a redistribution from lymph nodes to circulation [5]. Together with the effect on the immune system, ARV drugs suppress HIV viral replication, thus speeding up immune recovery. Notwithstanding prolonged suppression of viral load, approximately $15-30 \%$ of ARVtreated subjects lack CD4 T-cell increase [6]. A French study found that $17 \%$ of HIV-infected subjects initiating a protease inhibitor-based regimen had an increase of $<50$ CD 4 lymphocytes/ $\mu$ l after 6 months, despite attaining control of viral replication [7]. These subjects, who are defined as immunological nonresponders (INRs), have an increased risk of clinical progression to AIDS and death [8-10]. The ART Cohort Collaboration found that subjects with CD4 T cells $<200 / \mu \mathrm{l}$ 6 months after ART initiation were at a significantly higher risk of AIDS or death, compared with those who had CD4 T cells $>200 / \mu 1$ [11]. Other studies have also demonstrated that CD4 counts are related to overall mortality and incidence of non-AIDS cancers, even in subjects with CD4 lymphocytes $>350 / \mu l[12,13]$.

Moreover, the immune system could be diverted from its function against HIV by the presence of other comorbidities, such as chronic HCV infection [14] or underlying CMV infection [15].

Physicians caring for INR HIV-infected subjects struggle between two opposite options: leave ARV therapy as it is or start an experimental therapy in order to improve CD4 count and functionality. In general, one main concern is the long-standing duration of immune suppression. Patients do not show any change in CD4 cell counts and this leads to concern that some unexpected event is going to irreversibly compromise the health of the patient. Indeed, the cited literature demonstrates multiple risks. However, together with the many worries, we should study, research and understand the reasons why an expected phenomenon (i.e., the rise in CD4) is not occurring in the subjects we care for, notwithstanding optimal therapeutic adherence. Above all, we should consider two different standpoints. The first being immunopathogenetic: identification and comprehension of the mechanisms of immunological failure, and the second being clinical and therapeutic: screening for the main comorbidities and possibly proceeding with an intensification of anti-HIV treatment.

66

"...we should study, research and understand the reasons why an expected phenomenon ... is not occurring in the subjects we care for, notwithstanding optimal therapeutic adherence."

Some therapeutic trials for therapeutic intervention in INR have been completed (e.g., atorvastatin and hydroxychloroquine), while others are underway or have been proposed, such as rifaximin, IL-7, antifibrotic

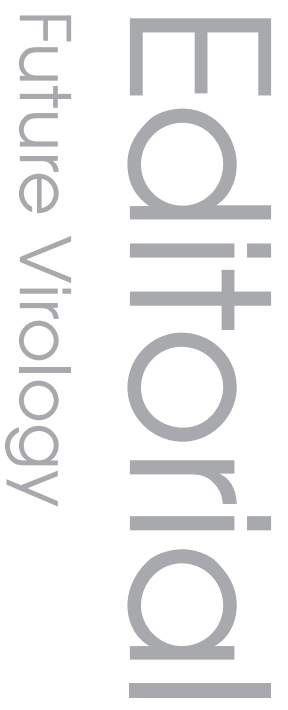

\section{Keywords}

- HIV infection $=$ IL-7 - immunological nonresponders $=$ maraviroc - treatment = tropism

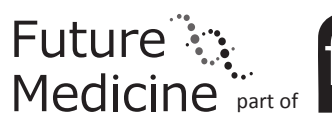


agents, maraviroc (MVC) and probiotics. In the past, IL-2 has been tested with the aim of increasing the number and function of CD4 lymphocytes, but this strategy did not produce long-term positive results [16]. Among the most promising novel strategies in this field, IL-7 seems to be useful in overcoming the impasse in the immune system in INR and clinical trials have been planned and completed [17]. Recently, MVC has been tested in three clinical trials. The first study, conducted by Peter Hunt at University of California, San Francisco (UCSF; CA, USA) did not indicate a significant increase in CD4 counts. Moreover, this trial indicated a rise in lymphocyte activation markers after the intensification with MVC [18]. The second study has been coordinated by the University of Milan (Italy). In this trial, MVC showed the ability to significantly increase IL-7 levels in plasma and the capacity to cause an increase in the number of CD4 lymphocyte, but only in subjects with baseline CD $4<200 / \mu \mathrm{l}$ after 48 weeks of intensification [19]. The third study, ACTG A5256, did not show an increase in CD4 T-cell counts of at least 20 cells/ $\mu \mathrm{l}$ after adding MVC to a virologically successful therapy for 24 weeks. In parallel, markers of immune activation and apoptosis decreased during MVC intensification, and this decline partially reversed after MVC discontinuation [20]. Overall, these results did not demonstrate the absolute benefit of adding MVC in HIV-infected subjects with an incomplete immunological recovery, although other immunomodulatory properties of MVC could be explored, such as the prevention of binding of $\beta$-chemokines and HIV gp120 to CCR5. This could probably explain the increase in CD4 lymphocytes in subjects with a dual/mixed (D/M)-tropic virus and an incomplete control of viral replication [21].

"...(the) results did not demonstrate the absolute benefit of adding maraviroc in HIV-infected subjects with an incomplete immunological recovery..."

If the blockade of CCR5 is not yet certain, another fascinating hypothesis is the administration of probiotics with the aim of preventing microbial translocation and hampering the increase in lipopolysaccharides plasma level seen in INR [22]. Of note, it has recently been shown that $\mathrm{CD} 4 \mathrm{~T}$ cells in the jejunum increase at a faster rate, CD4 T-cell frequencies in the colon are higher and activated CD4 T-cell frequencies in the colon are lower with ARV plus probiotic treatment in pigtail macaques acutely infected with SIVmac239 [23].

Although there are many new approaches to treat the immunological nonresponse to ARV drugs in HIV infection, the principal effort should be directed towards finding new HIV infections and treating earlier - according to the 'test-and-treat' strategy - in order to prevent a deeper depression of the immune system in both quantitative and qualitative terms.

\section{Financial \& competing interests disclosure}

$S$ Rusconi was the principal investigator in a Pfizer IIR (Investigator Initiated Research) investigating maraviroc in immunological nonresponders. The author has no other relevant affiliations or financial involvement with any organization or entity with a financial interest in or financial conflict with the subject matter or materials discussed in the manuscript apart from those disclosed.

No writing assistance was utilized in the production of this manuscript.

\section{References}

1. Brenchley JM, Douek DC. The mucosal barrier and immune activation in HIV pathogenesis. Curr. Opin. HIV AIDS 3, 356-361 (2008).

2. Lederman MM, Calabrese L, Funderburg NT et al. Immunologic failure despite suppressive antiretroviral therapy is related to activation and turnover of memory CD4 cells. J. Infect. Dis. 204, 1217-1226 (2011).

3. Armah KA, McGinnis K, Baker J et al. HIV status, burden of comorbid disease, and biomarkers of inflammation, altered coagulation, and monocyte activation. Clin. Infect. Dis. 55, 126-136 (2012).

4. Autran B, Carcelain G, Li TS et al. Positive effects of combined antiretroviral therapy on $\mathrm{CD}^{+} \mathrm{T}$ cell homeostasis and function in advanced HIV disease. Science 277, 112-116 (1997).

5. Bucy RP, Hockett RD, Derdeyn CA et al. Initial increase in blood $\mathrm{CD}^{+}$lymphocytes after HIV antiretroviral therapy reflects redistribution from lymphoid tissues. J. Clin. Invest. 103, 1391-1398 (1999).

6. Kelley CF, Kitchen CMR, Hunt PW et al. Incomplete peripheral $\mathrm{CD} 4^{+}$cell count restoration in $\mathrm{HIV}$-infected patients receiving long-term antiretroviral treatment. Clin. Infect. Dis. 48, 787-794 (2009).
7. Grabar S, Le Moing V, Goujard C et al. Clinical outcome of patients with HIV-1 infection according to immunologic and virologic response after 6 months of highly active antiretroviral therapy. Ann. Intern. Med. 133, 401-410 (2000).

8. Baker JV, Peng G, Rapkin J et al. $\mathrm{CD}^{+}{ }^{+}$count and risk of non-AIDS diseases following initial treatment for HIV infection. AIDS 22, 841-848 (2008).

9. Baker JV, Peng G, Rapkin J et al. Poor initial $\mathrm{CD} 4{ }^{+}$recovery with antiretroviral therapy prolongs immune depletion and increases risk for AIDS and non-AIDS diseases. J. Acquir. Immune Defic. Syndr. 48, 541-546 (2008).

10. Gazzola L, Tincati C, Bellistrì GM, Monforte A, Marchetti G. The absence of $\mathrm{CD}^{+}{ }^{+} \mathrm{T}$ cell count recovery despite receipt of virologically suppressive highly active antiretroviral therapy: clinical risk, immunological gaps, and therapeutic options. Clin. Infect. Dis. 48, 328-337 (2009).

11. Chene G, Sterne JA, May M et al. Prognostic importance of initial response in HIV-1 
infected patients starting potent antiretroviral therapy: analysis of prospective studies. Lancet 362, 679-686 (2003).

12. Lodwick RK, Sabin CA, Porter K et al.; Study Group on Death Rates at High CD 4 Count in Antiretroviral Naive Patients. Death rates in HIV-positive antiretroviralnaive patients with $\mathrm{CD} 4$ count greater than 350 cells per microL in Europe and North America: a pooled cohort observational study. Lancet 376, 340-345 (2010).

13. Bruyand M, Thiébaut R, Lawson-Ayayi S et al. Role of uncontrolled HIV RNA level and immunodeficiency in the occurrence of malignancy in HIV-infected patients during the combination antiretroviral therapy era: Agence Nationale de Recherche sur le Sida (ANRS) CO3 Aquitaine Cohort. Clin. Infect. Dis. 49, 1109-1116 (2009).

14. Kottilil S, Yan MY, Reitano KN et al. Human immunodeficiency virus and hepatitis $\mathrm{C}$ infections induce distinct immunologic imprints in peripheral mononuclear cells. Hepatology 50, 34-45 (2009).

15. Appay V, Fastenackels S, Katlama C et al. Old age and anti-cytomegalovirus immunity are associated with altered T-cell reconstitution in HIV-1-infected patients. AIDS 25, 1813-1822 (2011).

16. Abrams D, Levy Y, Losso MH et al. Interleukin-2 therapy in patients with HIV infection. N. Engl. J. Med. 361, 1548-1559 (2009).

17. Lévy Y, Sereti I, Tambussi G et al. Effects of recombinant human Interleukin 7 on T-cell recovery and thymic output in HIV-infected patients receiving antiretroviral therapy: results of a Phase I/IIa randomized, placebo-controlled, multicenter study. Clin. Infect. Dis. 55, 291-300 (2012).

18. Hunt P, Shulman N, Hayes T et al. Immunomodulatory effects of MVC intensification in HIV-infected individuals with incomplete $\mathrm{CD}^{+} \mathrm{T}$ cell recovery during suppressive ART. Presented at: $18 \mathrm{th}$ Conference on Retroviruses \& Opportunistic Infections. Boston, MA, USA, 27 February-2 March 2011 (Abstract 153LB).

19. Vitiello P, Merlini E, Colella E et al. Maraviroc as intensification strategy in immunological non-responder $\mathrm{HIV}^{+}$patients with virologic success on HAART. Presented at: 19th Conference on Retroviruses \& Opportunistic Infections. Seattle, WA, USA, 5-8 March 2012 (Abstract 630).
20. Wilkin TJ, Lalama CM, McKinnon J et al. A pilot trial of adding maraviroc to suppressive antiretroviral therapy for suboptimal $\mathrm{CD}^{+}{ }^{+} \mathrm{T}$-cell recovery despite sustained virologic suppression: ACTG A5256. J. Infect. Dis. 206, 534-542 (2012).

21. Saag M, Goodrich J, Fatkenheuer G et al. A double-blind, placebo-controlled trial of maraviroc in treatment-experienced patients infected with non-R5 HIV-1. J. Infect. Dis. 199, 1638-1647 (2009).

22. Brenchley J. Mechanisms underlying microbial translocation and novel therapeutic interventions to reduce microbial translocation-induced immune activation. Presented at: Italian Conference on AIDS and Retroviruses. Napoli, Italy, 10-12 June 2012.

23. Klatt N, Canary L, Sun X et al. Probiotic supplementation of ARV treatment during SIV infection of pigtail macaques results in enhanced GI tract $\mathrm{CD}^{+} \mathrm{T}$ cell frequency and immunological function. Presented at: 19th Conference on Retroviruses \& Opportunistic Infections. Seattle, WA, USA, 5-8 March 2012 (Abstract 95). 